\title{
Vehicle Control in Vehicle to Infrastructure (V2I) Environment
}

\author{
Shekhar Banerjee \\ Department of CSE \\ MS Ramaiah Institute of Technology \\ Bangalore, India
}

\author{
Divakar Harekal \\ Department of CSE \\ MS Ramaiah Institute of Technology \\ Bangalore, India
}

\begin{abstract}
The world is moving towards automation of everything, vehicles are also getting automated with driverless vehicles, and the infrastructure is being upgraded to facilitate them. Automation leads to less human time involved in a work and decrease the time taken by system to perform a task. To make the vehicles automated and have driverless features, the traffic monitoring system needs to have a very strong and well-built infrastructure to support the concept of Vehicle to Infrastructure (V2I) Technology. The objective is to improve the concept of traffic control system for vehicles. The traffic monitoring system should monitor each vehicle of a region and look for any traffic rule violation or any misbehavior that is being caused on the V2I environment. The traffic control should disable a vehicle automatically or charge fine remotely at that instant automatically if required, that violets traffic rules or misbehaves in the roadways. This paper has presented a concept of communication between automated vehicles and semi-automated vehicles and the infrastructure that could be implemented for smooth regulation of traffic, and also provides a few rules for governing such infrastructure.
\end{abstract}

\section{General Terms}

Vehicle Misbehavior, Traffic Violation, Vehicle to Everything Communication, Vehicle to Vehicle Communication, On Board Unit, Vehicle Control.

\section{Keywords}

Vehicle disable; vehicle to infrastructure; misbehavior; traffic light violation; V2X, V2I

\section{INTRODUCTION}

In some countries or some places it is seen that the traffic system is not good and are easier to get broken without any fear of penalty. In some places it is seen that the traffic system is not honest, and can be easily be broken by someone with the help of some bribe or some internal source. Every time the traffic rules and regulations are violated, human is involved. Either traffic rules are violated by human mistakes or carelessness driving or corruption encouraged by the system and the citizens.

So, these issues lead us to a question. Question such as, is there any way in which the society can get rid of these issues or not? If so, then, what is the solution? Will it be effective? Let us now discuss how to solve the problems that are faced as given above seen above.

To solve these problems first the traffic monitoring system needs to shift to a digital world of vehicles from manual vehicle control. Once the traffic monitoring system gets into the digital control of vehicles, the traffic monitoring system can move towards Vehicle to Vehicle (V2V) and Vehicle to Infrastructure (V2I) communication. On doing so the traffic monitoring system gets the interface to automate the vehicle driving on smart roadways. This is the first step in solving the problems.

There are multiple companies or organization that are involved in the research and development of V2I and V2V communication. They try to establish $\mathrm{V} 2 \mathrm{~V}$ communication within the vehicles, and V2I communication between the vehicles and the infrastructure that is available.

The main objective of this paper is to improve the infrastructure so as to improve the quality of the V2I communication and reduce misbehavior, carelessness and other human mistakes and crime.

\section{SOME TERMINOLOGIES}

\subsection{On Board Unit (OBU)}

An On Board Unit (OBU) is a module of a vehicle which has the capabilities of establishing the V2X communications and transmits Basic Safety Messages (BSMs) to other vehicles and other V2I units. As a whole it can be said that every vehicle which supports V2X communication and transmit BSMs, can be referred to as On Board Unit (OBU).

\subsection{Road Side Unit (RSU)}

A Road Side Unit (RSU) is the stationary infrastructure which supports the vehicles passes by providing different facilities such as traffic control lamp post, petrol pump, hospitals etc. Every RSU will extract alerts and events from the neighboring areas and communicate to the vehicles near to it.

\subsection{Dedicated Short Range Communication (DSRC)}

A Dedicated Short range Communication (DSRC) is a transceiver which is mainly used in $\mathrm{V} 2 \mathrm{~V}$ communication (802.11p IEEE standards). The DSRC transceiver broadcasts the vehicular data and status to its neighboring OBUs and RSUs, whereas, receiving all the data and status from the neighboring OBUs and infrastructure status and data from the neighboring RSUs, at the same time. Data TX and RX occurs at the same time in DSRC transceiver.

\subsection{Authentication Certification (AC)}

An Authentication Certificate (CRL) is a Certification of a vehicle which states that a vehicle is proper and well authenticated to get access to the smart roadways for proper $\mathrm{V} 2 \mathrm{X}$ communication while obeying the traffic rules and regulations and other protocols.

\subsection{Certification Revocation List (CRL)}

A Certificate Revocation List (CRL) is a list of vehicle IDs whose Authentic Certificate (AC) has been revoked due to some misbehavior act upon the environment they are moving. Once the AC is updated the vehicle IDs gets deleted from the 
CRL. If any neighboring vehicle misbehaves, the vehicle IDs get added to CRL, and the misbehaving vehicle's AC becomes invalid. The AC is updated on request for update.

\section{V2X COMMUNICATION}

$\mathrm{V} 2 \mathrm{X}$ communication means vehicle to everything communication. In V2X Communication the word "Everything" means communication between vehicles, infrastructures, and pedestrian (human, animals etc.). In a brief $\mathrm{V} 2 \mathrm{X}$ is a combination of Vehicle to Vehicle (V2V), Vehicle to Infrastructure (V2I) and Vehicle to Pedestrian (V2P), so let us discuss the different types of communication.

\subsection{V2V Communication}

In a V2V communication the vehicles communicate with the help of DSRC Transceiver. Where the transmission Tx and receive Rx occurs almost simultaneously. Every OBU listens to all the neighboring OBUs for BSMs (Basic Safety Messages) and other alerts which are being broadcasted from the neighboring OBU, and then once all the BSMs are received, the host OBU starts to broadcast its own BSMs for the other OBUs.

\subsection{V2I Communication}

Communication with the infrastructure is somewhat similar to V2V, using DSRC Transceiver. Every RSU listens to all the neighboring RSUs and OBUs for alerts which are being broadcasted over the air. The alerts and other information that the RSU broadcasts are from the neighboring OBUs and other sensors or the traffic management system. Once all the alerts are received, the RSUs start to broadcast its own alerts and messages to the other neighboring OBUs.

\subsection{V2P Communication}

The V2P communication is somewhat one side communication, where the V2P module has sensors such as RADAR (RAdio Detection And Ranging) or other sensors and the smartphone communication to detect the pedestrian. The V2P module searches for the different signals from smartphones and detect the human. It uses the RADAR and other sensors to detect human without smartphones and animals that is near to the OBU.

\section{TRAFFIC MONITORING SYSTEM}

The infrastructure of the roadways should be a well-defined and well established to support the $\mathrm{V} 2 \mathrm{~V}$ and V2I communication and interaction. The infrastructure should be very much secure, robust, reliable, trust worthy and quicksmart-responsive as human life is much more precious for them. So let us discuss how the traffic monitoring system can improve the traffic control system using the V2I Communication and interaction.

Companies such as Google, Delphi etc. are obsessed in improving V2V and V2I communication so as to increase the interaction between them, so as to make all the automobile vehicles automated and driverless. For doing such a big deed the traffic monitoring system needs infrastructure that can support the V2V and V2I interaction to be enhanced. As everything in becoming automated, the traffic management system has to be automated. Making an automated traffic management system, will result in less traffic protocols be broken, less corruption between the different system, can reduce crimes that occur in the roadways such as murder by running over lives. On

Let us see how to automate the traffic management for the betterment of the V2I interaction:-

\subsection{Traffic Light Violation}

We will have multiple sensors to detect every movement of every OBU that passes by a traffic signal (RSU). Let us look for the movements which are violating the red light of the traffic signal. On such violation the traffic management system can provide a warning to the $\mathrm{OBU}$, register a fine to for the OBU owner against the vehicle number and force the owner to be interrogated for the action, within a small span of time, say a week or 10 days, else the fine gets multiplied. On interrogation if the owner or driver claims to have not broken the protocols then the vehicle automated system should be examined for the reason of violation and check for the logs. If in one day any OBU violets rules multiple times the traffic monitoring system can automate the traffic management system to disable the vehicle and prevent the vehicle from moving further. On disabling, the management system gets a call for manual inspection. This is how the traffic management system can control the traffic red light violation and get away from manual inspection directly by human. Let us see an algorithm that might solve the problem.

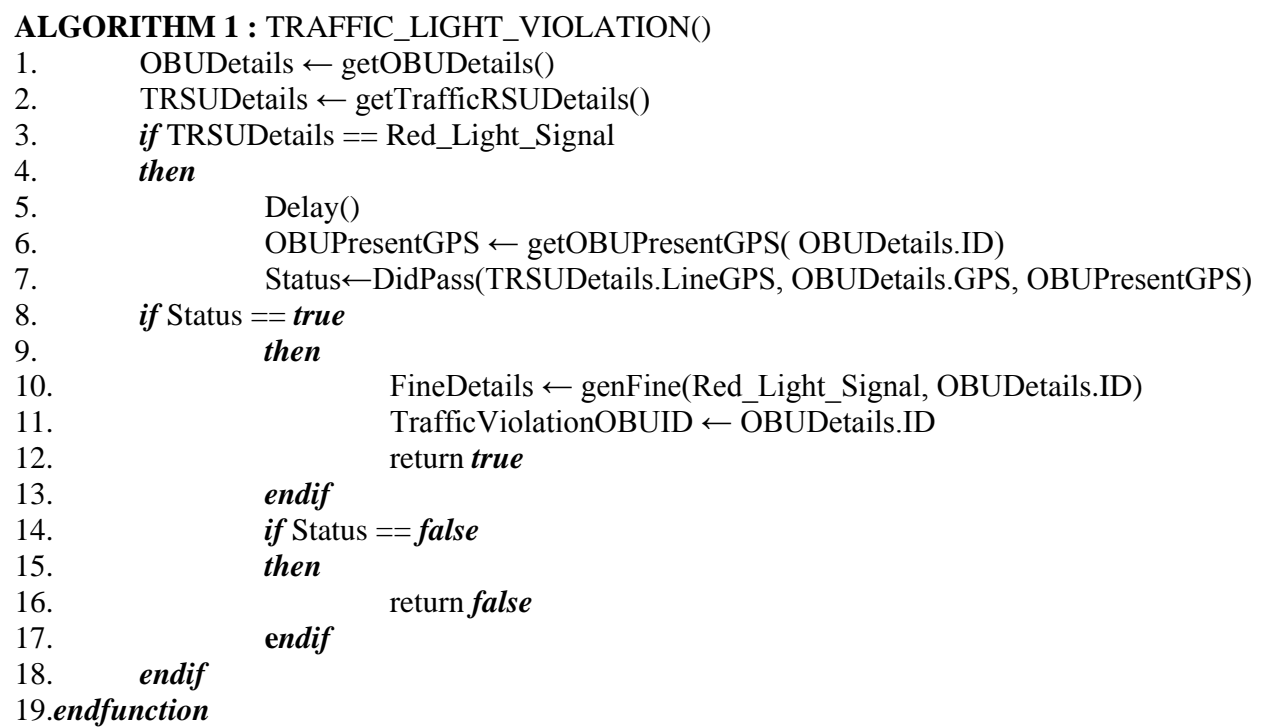


Where,

1. getOBUDetail() gets all the details of the OBU or Vehicle.

2. getTrafficRSUDetails() gets all the traffic details.

3. Red_Light_Signal is a Traffic signal number which denots the red signal.

4. getOBUPresentGPS() gets the present GPS of the given OBU.

5. DidPass() function checks whether the OBU passed the Traffic Line GPS or not.

6. genFine() on true violation of red light, generates a fine at the traffic management system against the OBUID

\subsection{Misbehavior control}

Vehicle misbehavior is one which is very difficult to detect as it may not be sensed by any sensor. It can however be detected by logic and algorithm for different scenarios. For an instance, a false road block signal is misbehavior. The Infrastructure does not have any sensors to detect whether an OBU is broadcasting a false road block signal or a true one. So, an algorithm might be helpful in detecting the false alerts which will not be detected by the sensors. In this false road block alert the RSUs should start tracking the OBU that broadcasted the road block alert until multiple OBUs send the same alert at the same location or the OBU that gave the alert, passes through the claimed road block location in no time. If multiple OBUs give the same alert for the road block then their might me a real road block, on the other case if the OBU that claimed the road block alert passes through that location, the OBU must have misbehaved and given a false alert so as to reduce other OBUs to move away from its path or take other path. This is how it can make logic and algorithm for the misbehavior that cannot be sensed by any sensors. Let us see an algorithm which might depict the logic to detect whether a road block alert is real or not.

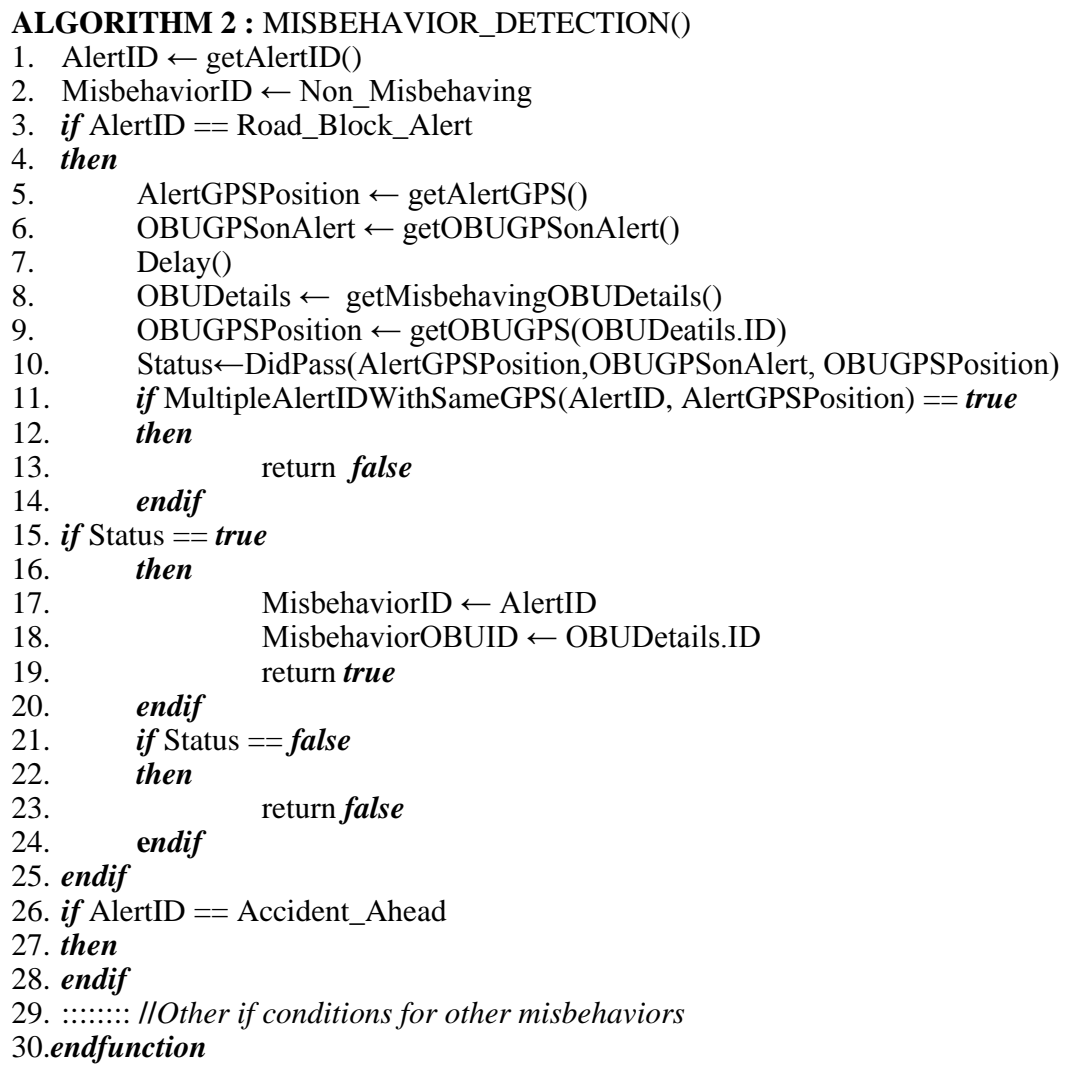

Where,

1. Road_Block_Alert is the alert number which denotes the road block alert.

2. getAlertID() function gets the alert ID.

3. getAlertGPS() function gets the GPS location where the alert occurred.

4. getOBUGPSonAlert() function gets the GPS location of the OBU where it triggered the alert.

5. Delay() function provides a delay for the OBU to travel ahead in case of misbehavior.

6. getMisbehavingOBUDetail() gets all the details of the OBU or Vehicle.

7. getOBUGPS() function which returns the immediate GPS of the given OBU.

8. DidPass() function checks whether the OBU passed the road block GPS or not. 
9. MultipleAlertIDWithSameGPS() function checks for whether there are multiple same type of alert are being triggered by multiple OBUs at the same GPS location or not.

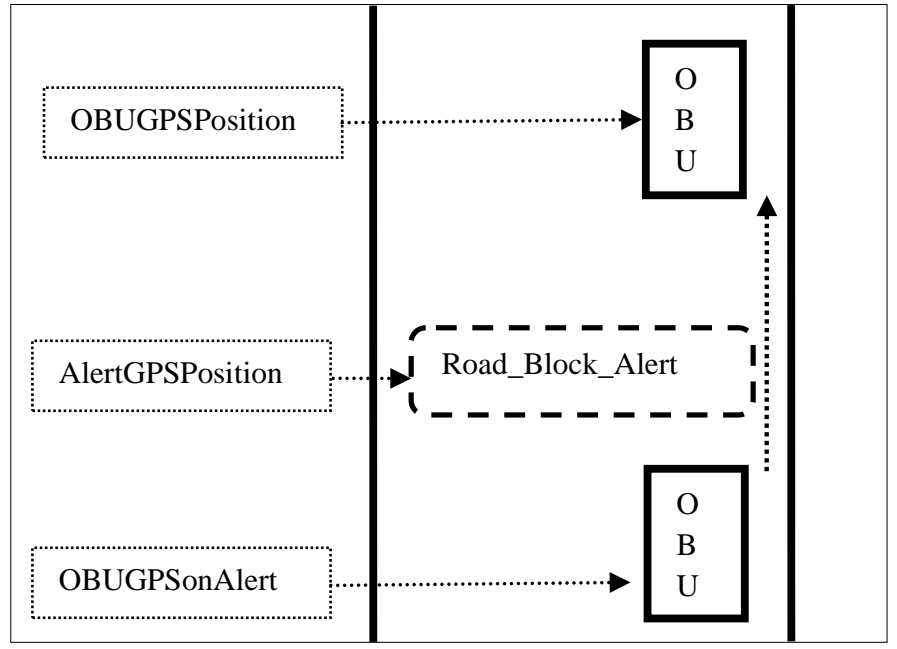

Figure 1: Road_Block_Alert Misbehavior $=$ true

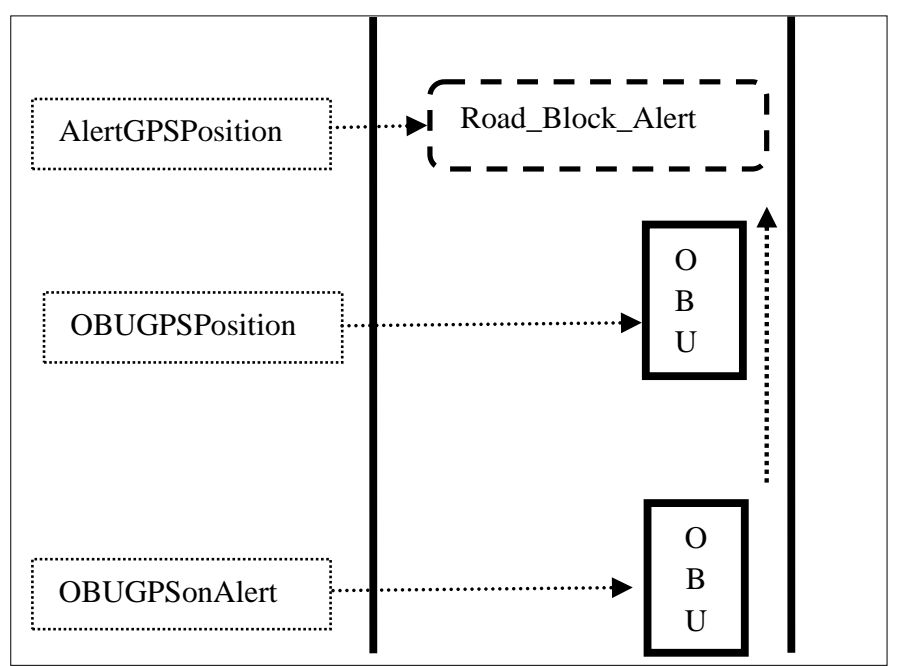

Figure 2: Road_Block_Alert Misbehavior = false

As in the Figure 1 it can be seen that misbehavior created by an OBU as the OBU alerts a wrong Road_Block_Alert and the passes the road block position. This signifies that the OBU was misbehaving. On the other hand the Figure 2 shows the OBU giving a Road_Block_Alert, but however it was unable to pass the road block position. The second scenario proves that that the vehicle might not have misbehaved, there might be a chance for a roadblock. In the case of second scenario (Figure 2) the Traffic management needs to wait and check for multiple Road_Block_Alert at that same GPS location for confirmation.

\subsection{DidPass() Function}

The DidPass() function call is very simple to calculate and decide whether a vehicle has passed the particular GPS location or not. This function will have three arguments, which will be enough to arrive to a conclusion whether a vehicle has passed a particular point or not, hence arrive to a conclusion about the traffic violation or misbehavior. The function returns the Boolean status of a vehicle, whether it has passed or not. The prototype of the function should be as follows:

Boolean DidPass(<PointOfPass $>$, <OBUPosWhileAlert/ Event $>$, <OBUPosAfterDelay $>$ );

Where,

1. PointOfPass: GPS of the Alert or Event to be observed for passing.

2. OBUPosWhileAlert/Event: Position of the OBU at the time of event or alert.

3. OBUPosAfterDelay: Position of the OBU after some delay, which will help us determine the status.

Let us see the algorithm for the DidPass() function, which will help approximately us find whether the OBU is passing the alert/event specific GPS. 


\section{ALGORITHM 3 : DIDPASS (PointGPSOfPass, OBUPosWhileAlert/Event, OBU PosAfterDelay)}

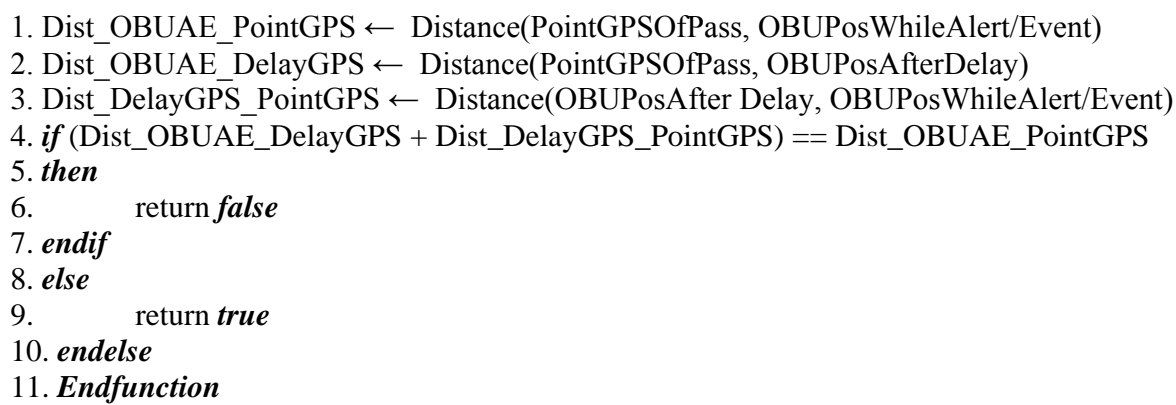

Whether an OBU is doing any kind of misbehavior or traffic rules and regulation violation or anything critical to the roadways are subjected to revocation of the AC (Authentication Certificate), and hence the OBUID will be added to the CRL (Certification Revocation List). Once the certificate is revoked, the OBU will have to request for the updated $\mathrm{AC}$ to be downloaded. If the $\mathrm{AC}$ is not updated the OBUs will not be able to communicate, the V2X communication gets disabled and hence it becomes a vehicle without the V2X capabilities.

\section{VEHICLE CONTROL ON MISBEHAVIOR}

As it is seen that people often tend to violet traffic rules and regulation, or bribe the traffic police if they are caught for violating it, the traffic monitoring system can make these things stop if the traffic management system is made automated. In cases of dangerous situations as it is required for a vehicle to be stopped and kept on hold manually. Similarly a system that can stop an OBU on the event of misbehavior or traffic violation or rash driving is needed. On the other hand if the OBUs are automated, it may experience a situation where some hardware or software might get damaged and hence the OBUs start to misbehave. In such a huge massacre situations the roadways might get compromised and hence be prone to multiple accidents. Let us see some situations when we can experience a situation which has only one solution and that is to disable the vehicle from moving further.

5.1. Due to rain water leakage there might be a possibility that the V2X module or some hardware got destroyed. On such situations the OBUs might be misbehaving on the roadways without any manual human mistakes. It is better for the traffic management system to disable the OBU and gradually stop, for further movement on the roadways and prevent the OBU from making accidents.

5.2. In cases of crime related issues, it is seen that the vehicles (OBU) tends to have a rash driving to get rid of police or other people while on a chase. On such situations it will be a good practice to disable the OBU. However the criminal OBU ALGORITHM 4: DISABLE_OBU ()

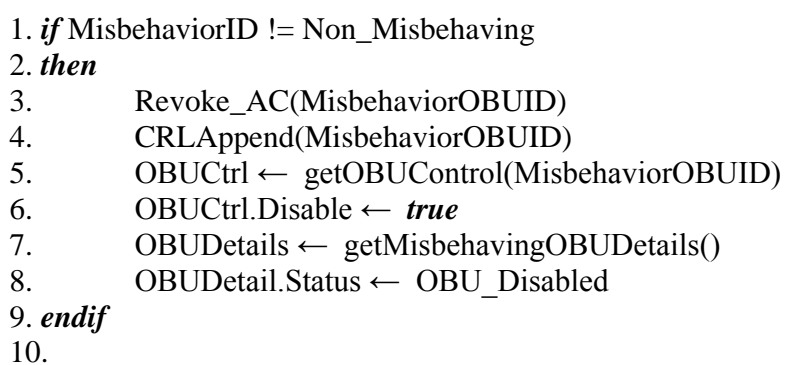

11. if Traffic ViolationOBUID $!=0$ disablement be either automatic or manual, and lock the door as well so as to stop the criminals from fleeing.

5.3. It is sometimes seen that the OBU experiences a dangerous situation of break fail die to some reasons. So, during break fail the driver can disable the OBU, on which the OBU gradually slows down till it stops and hence save the people and other OBUs and infrastructures. This disablement will be triggered by the people sitting inside it.

5.4. Illegal hackers are very dangerous, if they somehow get hold of the traffic management system and gets the control of OBUs and RSUs, the situation gets very risky. If this kind is experienced by the people then the people inside the vehicle can disable the vehicle which gradually slows down till it stops.

The traffic management system, the roadway infrastructures and the automotive industries must take care of the manual and automated disable triggering from both ends i.e. from vehicles and the traffic management system or other infrastructures. On an event of vehicle disable the vehicle slows down gradually until it stops, and once it stops the traffic management system should send some manual inspection team to inspect on the vehicle for the fault and solutions for it. Manual inspection is better so as to arrive at a safer and proper solution to the issue it had faced.

Vehicle disable does not mean that the V2X module will be disabled for V2X communication. Vehicle disablement will simply trigger the V2X module to stop the engine instead of running and which will release all the pressure from the IC engine, which will gradually slow down and stop the vehicle. However the V2X communication still be on with high alert for the RSUs and the traffic management system, as it is required for everyone to know that the vehicle is facing a major problem.

Let see and algorithm which might help us build a module that will be able to disable a vehicle (OBU) for further movement. 


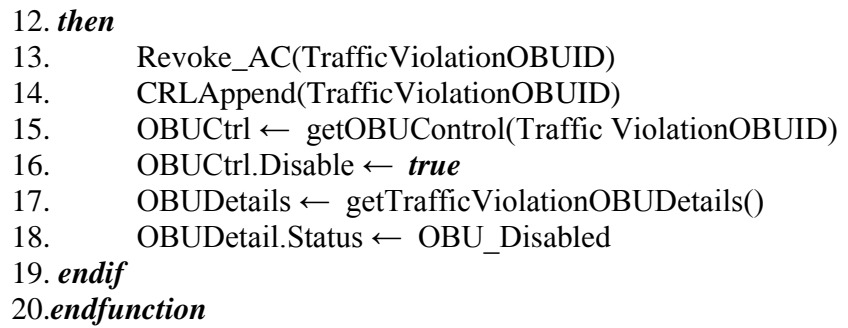

Where,

1. Revoke_AC() function revokes the Authentication Certificate.

2. CRLAppend() function appends the OBUID to the Certificate Revocation List which is misbehaving or violating traffic signals.

3. getOBUControl() fetches the control of the OBU which is misbehaving or violating traffic signals, so as to override the control of the OBU to disable it.

4. getMisbehavingOBUDetails() function fetches the details of the Misbehaving vehicle (OBU).

5. getTrafficViolationOBUDetails() function fetches the details of the vehicle (OBU) which is violating traffic signals.

As an example of how the traffic monitoring system could disable an OBU on the situation of misbehavior or traffic violation. The traffic monitoring system can also do the same for other misbehavior or other traffic rules and regulation violation. The traffic monitoring system can do the OBU disablement for the safer roadways which can cause huge accidents.

\section{CONCLUSION}

In today's world the whole automotive industry is involved in the automation of all high end vehicles and implementation of V2X communication in all vehicles. With this applications a strong infrastructure and a strong traffic management system to support this application is needed. To make the traffic management system easier the traffic monitoring system needs to follow the concept of vehicle disabling. It not only stops human from getting into dangerous situations but also prevents them from crimes such as hit and run, murder, traffic rule and regulation violation etc. and many more.

\section{ACKNOWLEDGMENTS}

The successful completion of any task would be incomplete without the mention of my guide Dr. Divakar Harekal, who made it possible because "Success is the abstract of hard work \& perseverance, but steadfast of all is encouraging guidance". So, I acknowledge his guidance and encouragement served as a beacon light \& crowned my effort with success. Finally I would like to thank all my friends, parents who have been with me with their valuable suggestions in making this Industrial Training a grand success.

\section{REFERENCES}

[1] Daxin Tian, Hao Luo, Jianshan Zhou, Yunpeng Wang, Guizhen $\mathrm{Yu}$ and HaiyingXia, "A Selp-adaptive V2V Communication System with DSRC," IEEE International Conference on Green Computing and IEEE Internet of Things and IEEE Cyber, Physical and Social Computing, ISBN: 978-0-7695-5046-6, August 2013, pp. 15281532 .

[2] Peppino Fazio, Floriano De Rango and Andrea Lupia, "A New Application for Enhancing VANET Services in Emergency Situations Using the WAVE/802.11p Standard, " IEEE Conference on International Federation on Information Processing, ISBN: 978-14799-0543-0 , Nov 2013.

[3] Kevyan Ansari, Charles Wang, Lei Wang and Yanming Feng, "Vehicle-to-Vehicle Real-Time Relative Positioning Using 5.9 GHz DSRC Media," Vehicular Technology Conference (VTC Fall), IEEE $78^{\text {th }}$ Conference, Supported by Commonwealth of Australia through the Cooperative Research Centre for Advanced Automotive Technology (AutoCRC) project C3-23, ISBN: 978-1-4673-6187-3, September 2013.

[4] Vladimir Grigoryev, Igor Khvorov, Yury Raspaev and Elena Grigoreva, "Intelligent Transportation Systems: Techno-Economic Comparison of Dedicated UHF, DSRC, Wi-Fi and LTE Access Networks," Case study of St. Petersburg, Russia, Conference of Telecommunication, Media and Internet TechnoEconomics (CTTE), ISBN: 978-1-4799-8238-7, IEEE, November 2015.

[5] Sushmita Ruj, Marcos Antonio Cavenaghi, Zhen Huang, Amiya Nayak, and Ivan Stojmenovic, "Data-centric Misbehavior Detection in VANETs," IEEE Vehicular Technology Conference (VTC Fall), ISBN: 978-1-42448327-3, September 2011.

[6] Moni Naor and Kobbi Nissim, "Certificate Revocation and Certificate Update," IEEE Journal on Selected Areas in Communications, ISSN: 0733-8716, August 2002. 